\title{
Criminologie
}

\section{La punition commune du crime : la prison et l'amende à Montréal de 1845 à 1913}

\section{Pierre Tremblay et Guy Therriault}

Volume 18, numéro 1, 1985

L'histoire du contrôle social

URI : https://id.erudit.org/iderudit/017207ar

DOI : https://doi.org/10.7202/017207ar

Aller au sommaire du numéro

Éditeur(s)

Les Presses de l'Université de Montréal

ISSN

0316-0041 (imprimé)

1492-1367 (numérique)

Découvrir la revue

Citer cet article

Tremblay, P. \& Therriault, G. (1985). La punition commune du crime : la prison et l'amende à Montréal de 1845 à 1913. Criminologie, 18(1), 43-76.

https://doi.org/10.7202/017207ar
Résumé de l'article

Common jails "produce" more punishment than either penitentiaries or reformatories for juvenile delinquents. Students of incarceration, however, have hitherto overlooked the significance of what could be called "petty" or minor punishment. Montreal's penal archives (1845-1913) have been systematically analyzed so as to permit a preliminary theory of such petty punishment institutions, their junction in the general penal economy and their evolution over time. 


\section{LA PUNITION COMMUNE DU CRIME : LA PRISON ET L'AMENDE À MONTRÉAL DE 1845 À 1913* \\ Pierre Tremblay** \\ Guy Therriault***}

Common jails «produce» more punishment than either penitentiaries or reformatories for juvenile delinquents. Students of incarceration, however, have hitherto overlooked the significance of what could be called «petty» or minor punishment. Montreal's penal archives (1845-1913) have been systematically analyzed so as to permit a preliminary theory of such petty punishment institutions, their function in the general penal economy and their evolution over time.

\section{INTRODUCTION}

La punition du crime ne s'organise pas autour d'une délimitation qualitative des pratiques criminelles : il n'existe pas d'institutions pénales propres aux voleurs, aux fraudeurs ou aux meurtriers. C'est plutôt la gravité relative du crime qui différencie une institution pénale d'une autre. Le paysage pénal moderne apparaît de loin assez monotone. À la délinquance banale, aux désordres ponctuels de la vie publique, on inflige une amende légère ou une détention brève; à la criminalité grave, persistante, majeure, on oppose l'incarcération prolongée, "pénitentiaire», et parfois même la peine de mort. Deux sortes d'institutions, deux manières de punir selon que le crime apparaît grave ou bénin. Cette structure à deux paliers peut se compliquer lorsque l'appréciation de la gravité d'un crime dépendra de l'âge du délinquant, de son état mental ou de sa «dangerosité» relative. On verra apparaître alors des institutions punitives spécifiques aux délinquants juvéniles, aux *criminels lunatiques» (une expression de l'époque), aux toxicomanes et l'édifice pénitentiaire deviendra lui-même un réseau plus subtil d'établissements subordonnés à des contraintes «sécuritaires» d'intensité variable. Mais la gravité relative du délit demeure pourtant un critère fondamental de la justice criminelle du $19^{\mathrm{e}}$ siècle et servira de base de discussion.

* Cête recherche a bénéficié d'une subvention du Conseil de recherches en sciences humaines du Canada et de la collaboration des Archives nationales du Québec à Montréal. L'article résume une partie d'une thèse de doctorat consacrée à l'évolution du comportement pénal de l'ensernble de la justice criminelle mortréalaise du $19^{\mathrm{e}}$ siècle. On trouvera dans cette thèse le détail des données utilisées. Ces données ont fait l'objet d'une programmation accessible, sur demande, aux chercheurs qui s'y intéresseraient.

** M. Pierre Tremblay, attaché de recherche, Centre international de criminologie comparée, Université de Montréal.

*** M. Guy Therriault, École de criminologie, Université de Montréal. 
Appelons «commune» la punition qu'on impose à la petite délinquance banale puisque la prison qui la châtie a longtemps été officiellement qualifiée de prison "commune» pour la distinguer de l'incarcération pénitentiaire. La peine commune présente un profil caractéristique : on l'utilise très souvent (un grand nombre d'individus en subissent l'expérience) mais son intensité est à chaque fois très faible (le montant de l'amende est généralement peu élevé, la durée de la détention ne dépasse pas en moyenne un à deux mois). Elle semble de prime abord moins fascinante que la peine de mort, moins significative que l'incarcération pénitentiaire. Ce qui est vrai de la punition ordinaire, est également vrai de la délinquance banale ou des délinquants occasionnels qu'elle prend pour cible. La littérature académique s'est surtout intéressée, en fait, au registre de la peine majeure (et de la délinquance grave). D'où l'objectif de cet article : reconstituer l'évolution et la structure de cette peine mineure que la société montréalaise infligea à ses délinquants de 1845 à 1913.

\section{LES DONNÉES}

Les statistiques pénales de l'époque n'indiquent pas le plus souvent les modifications apportées aux règles de dénombrement qu'elles utilisent. À partir de 1899 , le décompte dans les registres d'écrou et dans les statistiques se fait par chef d'accusation et non plus par individu condamné. Un individu condamné pour deux délits sera compté deux fois. $\mathrm{Si}$ on inclut généralement parmi les condamnés ceux qui ne paient l'amende à laquelle ils avaient été condamnés qu'après avoir purgé une partie de leur incarcération, il arrive également qu'on les exclut (comme en 1889-1892). Deux modifications subreptices qui peuvent inciter à interpréter une hausse ou une baisse, à chaque fois fallacieuse, du taux de condamnation à la peine commune.

Contrairement aux registres d'écrou, le contenu des statistiques pénales est beaucoup plus fluctuant. Ce n'est qu'en 1870 que l'on s'avise de traiter les registres d'écrou de manière à ventiler les condamnés selon la durée de leur incarcération. Malgré l'existence de registres en bon ordre aucune statistique complète n'est disponible pour la période de 1854 à 1868 . Il faut se contenter d'un bilan récapitulatif présenté lors du rapport annuel de 1869 des inspecteurs (dans les documents sessionnels). Par chance ce bilan peut être contrôlé en certains points grâce à certaines statistiques incluses dans les documents parlementaires du régime préconfédéral. On s'aperçoit alors que les documents parlementaires des années 1865 et 1866 contredisent violemment les chiffres présentés par les documents sessionnels de l'année 1869. 
Finalement, les statistiques expriment mais ne contrôlent pas l'impact du flux des innovations et des préoccupations publiques. Ce n'est que durant les années 1890 que l'on se préoccupe de la manière dont les individus «sortent de prison», cette préoccupation se matérialisant en une consignation différenciée des individus libérés par acquittement (à partir de 1890), sans procès (1895), sous caution (1890) ou grâce à un "permis de libération" (1899). Mais faute de repères il est difficile de savoir si la catégorie "sorties de prison» est bien homogène avant et après 1890 .

Pour apprécier la fiabilité des statistiques pénales de l'époque on a comparé les registres d'écrou aux statistiques pénales relativement au nombre de personnes admises à la prison commune de 1845 à 1913 : 1) les statistiques publiques sous-évaluent généralement les données livrées par les registres d'écrou : il est très rare qu'on surestime la population carcérale, mais fréquent qu'on la sous-estime; 2) cette sousestimation n'est pas systématique, elle varie dans le temps : presque inexistante de 1845 à 1876 , elle est très élevée de 1865 à 1875 , mais beaucoup plus faible de 1877 à $1913 ; 3$ ) l'incertitude que suscitent les statistiques ne varie pas seulement dans le temps, mais aussi d'une catégorie à l'autre : certaines parties sont beaucoup plus fiables que d'autres, certaines institutions pénales produisent des résumés plus exacts que d'autres.

C'est en découvrant, progressivement, la clarté obscure des statistiques pénales et la richesse brute des registres d'écrou qu'elles résument qu'un certain nombre de règles de prudence se sont imposées. Il fallait tout d'abord se baser sur les registres d'écrou comme source première et limiter les statistiques à leur rôle d'appoint (source secondaire). Les registres présentant une quantité d'informations beaucoup plus écrasante que les statistiques pénales, la tentation de procéder à des échantillonnages divers semblait attrayante : analyser les registres tous les cinq ans ou les traiter annuellement mais se limiter à un certain quota de pages sélectionnées au hasard. Mais la première solution rendait impossible une analyse développée de séries chronologiques et la seconde ne convenait pas au rythme de travail spécifique aux tribunaux criminels. Les deux solutions partageaient, en outre, l'inconvénient de rendre incertain le repérage et la compréhension d'une transformation subite des règles de classement et de dénombrement. D'où la deuxième prudence : analyser exhaustivement des registres d'écrou et procéder à un recomptage $a b$ novo de toutes les données. Finalement, un certain nombre de définitions ont été adoptées pour s'assurer de l'homogénéité des catégories du début à la fin de la série : 1) les personnes «admises» à la prison 
commune regroupent les prévenus, les transférés et les condamnés; 2) on appelle transférés les individus qui upassent par la prison de Montréal» mais qui cheminent vers d'autres destinations (l'asile, le pénitencier, d'autres prisons en dehors de Montréal); 3) lorsqu'un individu est transféré dans une prison commune à l'extérieur de Montréal, il fera partie du groupe des individus condamnés à Montréal mais pas dans les autres cas; 4) prévenu désigne tout individu détenu (incarcéré) à la prison commune mais qui n'est pas condamné comme tel à purger unepeine d'emprisonnement commun; 5) lorsqu'une personne ne paie l'amende à laquelle elle avait été condamnée qu'après avoir purgé une partie, même minime, de son incarcération, nous l'insérons dans la cohorte des "condamnés à la prison commune»; 6) de 1899 à 1913, les individus accusés de plusieurs délits ne sont cependant comptés qu'une seule fois ; 7) une informatisation complète des registres d'écrou aurait seule permis l'évaluation de l'importance relative des "récidivistes" de la prison commune et comme, de plus, l'identification au $19^{\mathrm{e}}$ siècle d'un "récidiviste» demeurait très incertaine (en témoigne l'œuvre d'Alphonse de Bertillon), cet aspect du problème a été exclu de l'analyse bien qu'une variation dans l'intensité de la récidive puisse modifier artificiellement l'estimation du nombre d'individus condamnés à la prison commune; 8) les prisons communes spécifiquement destinées aux femmes n'ont été ouvertes qu'au début des années 1880 et celles-ci dès lors produisent leurs propres registres, également bien conservés : la prison commune montréalaise ne désigne donc pas dans cette recherche un établissement en particulier, mais selon les périodes l'ensemble des établissements qui dans ce territoire infligent la peine commune.

\section{MESURER LA PEINE COMMUNE}

Combien d'individus une société punit-elle? Quelle a été l'intensité de la peine infligée? Quelle quantité globale de peine est produite pour réprimer le crime et maintenir l'ordre public? Ces questions sont banales et on devrait pouvoir y répondre sans difficulté. Seule pourtant la philosophie pénale se réfere à la punition du crime comme une grandeur globale déterminée. Il est très difficile de trouver dans les statistiques officielles ou même dans la littérature spécialisée des réponses nettes à ces questions. On se limite à calculer la fréquence avec laquelle on punit et on néglige de tenir compte de son intensité variable. On évalue l'impact d'une mesure pénale mais sans considérer son rôle dans l'économie générale de la punition. On compare les fluctuations annuelles de la punitivité produite par la justice criminelle mais en omettant de situer ces variations ponctuelles dans une série chronologique étalée. 
La première opération (pour en arriver à mesurer le taux de punition) fut de déterminer l'évolution annuelle de la fréquence avec laquelle la justice pénale montréalaise inflige la punition commune. Pour suivre l'évolution de cette activité punitive, il était préférable d'isoler, dans la masse indifférenciée des «admissions» annuelles à la prison la cohorte de ceux qui y étaient condamnés, de la séparer, par conséquent, des prévenus (qui eux ne sont pas condamnés) et de la majorité des «transférés" (qui eux ne sont pas des condamnés «communs»). La fréquence de la peine commune désigne donc l'ensemble des individus qui y sont spécifiquement condamnés.

C'est plutôt la fréquence relative, le taux (par $10000 \mathrm{~h}$.) de condamnation à la peine commune qui apparaît comme la mesure adéquate. Les recensements décennaux de la ville de Montréal ont été utilisés à cette fin (Linteau et coll., 1979; Bernard et coll., 1975; Hamelin et Roby, 1971). Les taux calculés se réfêrent toujours à l'évolution démographique de la ville de Montréal et non pas de la «cité» ou de la "région» de Montréal. On obtient alors, par interpolation, l'accroissement annuel de la population de la ville de Montréal de 1845 à 1913. La figure 1 présente le résultat de cette première opération : l'évolution de la fréquence relative de la peine commune, c'est-à-dire du taux de condamnation par 10000 habitants à la prison ordinaire.

La deuxième opération fut de déterminer l'évolution de la sévérité des tribunaux criminels en matière de peine commune. La distribution annuelle des sentences infligées a été recalculée pour toute la période. Après avoir établi, suite à une comparaison avec les registres d'écrou, que les statistiques relatives aux sentences étaient fiables pour les années 1892 à 1896 et 1902 à 1912, on obtint un barème qui simplifia le dénombrement. Une sentence de moins d'un mois à moins de trois mois signifie en moyenne une sentence de 2,4 mois; une peine de quatre à six mois correspond à une sentence moyenne de 5,3 mois; finalement une sentence de plus de six mois correspond à une détention de 11,2 mois. Un dénombrement analogue, basé sur les statistiques moins fiables des années 1873,1876 et 1879 à 1891 aboutit au même barème. Cette répartition annuelle des sentences servit de base au calcul de la durée moyenne des sentences infligées par les tribunaux. La figure 2 présente l'évolution, exprimée en mois de détention, de la dureté relative de la peine commune à Montréal de 1845 à 1913.

Connaissant alors la fréquence relative de la peine commune d'une part et la sévérité annuelle des tribunaux d'autre part, il devient possible de calculer la quantité annuelle de punitions infligées. La première 
Fig. 1 - Taux de personnes condamnées à la prison commune à Montréal de 1845 à 1913 (par 10000 h.).

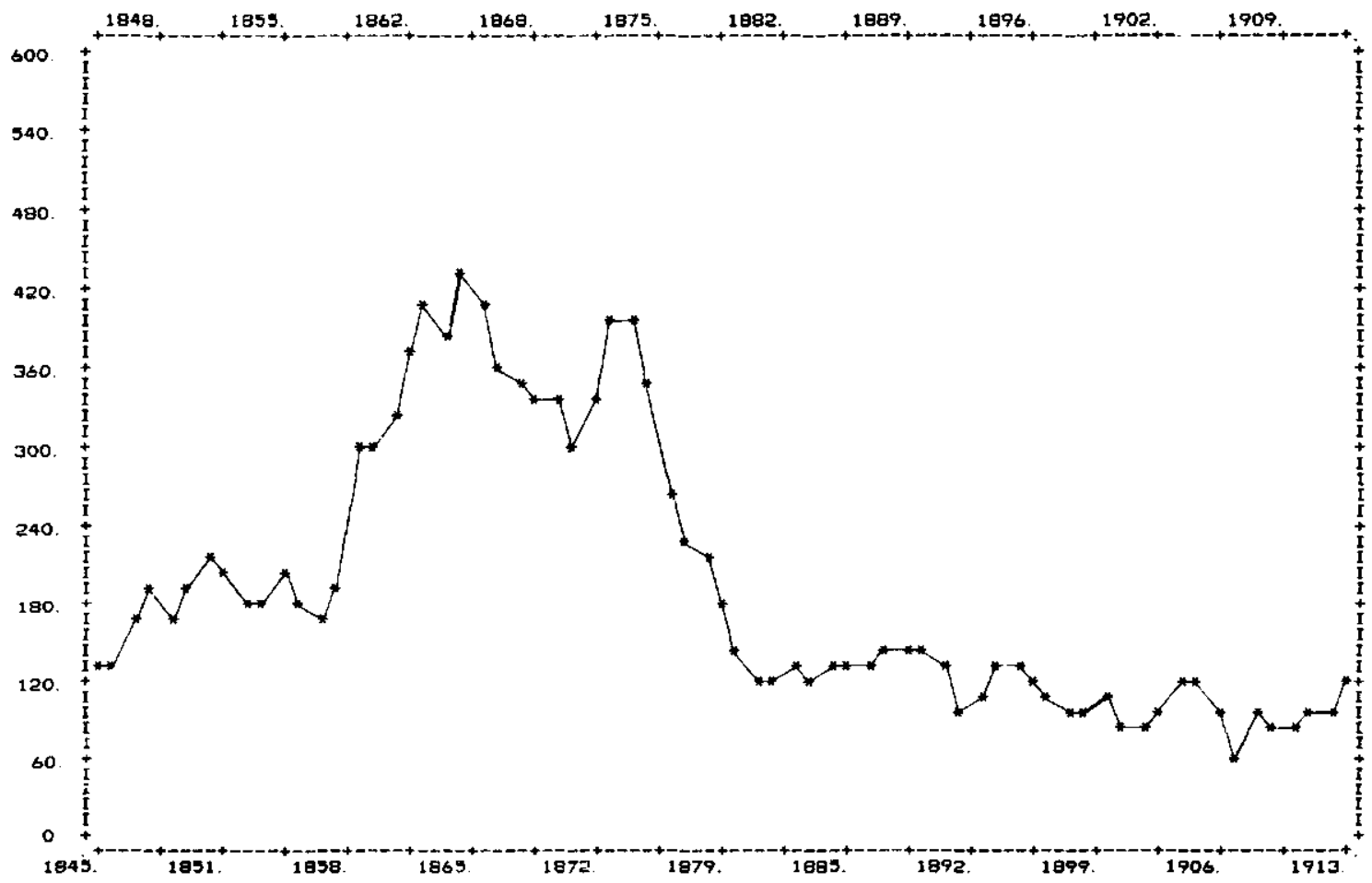


Fig. 2 - Sévérité annuelle moyenne des sentences purgées à la prison commune à Montréal de 1845 à 1913 (en mois).

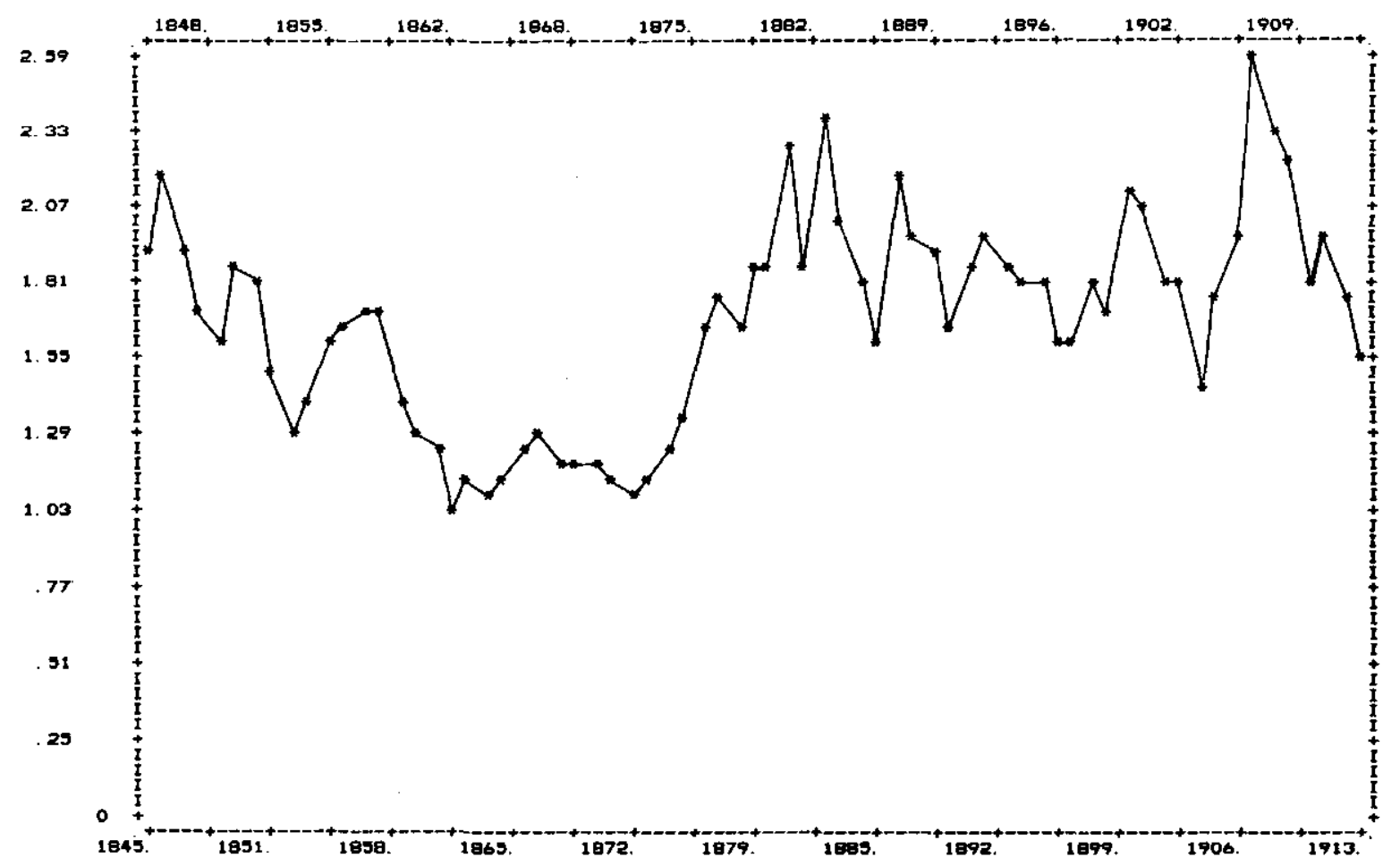


mesure étant exprimée en taux par $10000 \mathrm{~h}$. et la seconde en mois, ce quantum que l'on obtient en multipliant la première par la seconde s'exprime en "hommes/mois".

Il existe, bien entendu, une manière plus rapide et très populaire de procéder : l'examen des variations de la population carcérale annuelle. Si la sévérité des tribunaux varie, cette population fluctuera puisque celleci dépend de la durée moyenne des sentences. Si la fréquence relative de la peine varie, la population carcérale en sera affectée en conséquence puisqu'elle dépend aussi du nombre d'individus punis. Mais le taux de la population carcérale est une mesure moins précise parce qu'elle mesure l'effet complexe de plusieurs processus non différenciés: sévérité de la peine durant l'année en cours $e t$ les années antérieures (surtout au niveau de la peine pénitentiaire), fréquence relative des condamnations durant l'année en cours et les années antérieures, fluctuations enfin des libérations anticipées durant l'année en cours $e t$ les années antérieures. Le taux per capita de la population emprisonnée (qui distingue rarement, de surcroît, la détention des prévenus et la punition des condamnés) apparaît donc comme la moyenne mobile des conséquences du taux de punition produite par la justice criminelle durant une période qui déborde largement à chaque fois l'année de référence. La figure 3 présente le résultat de toute la démarche : l'évolution du taux de punition commune infligée par la justice criminelle montréalaise durant la deuxième moitié du $19^{\mathrm{e}}$ siècle.

\section{ÉVOLUTION DE LA PUNITION COMMUNE : UN CONSTAT PRÉLIMINAIRE}

De manière générale lorsqu'on punit plus souvent, on punit moins durement, et inversement. La corrélation est forte et négative $(r=-, 82)$. Deux points tournants peuvent être repérés. À partir de 1859 on punit plus souvent mais moins longtemps. À la fin des années 1870 , par contre, la situation inverse se produit : on punit beaucoup moins souvent mais plus durement. Même si la fréquence relative et la sévérité variable de la peine commune varient en sens inverse, elles ne s'annulent pas complètement.

Ainsi, de 1860 à 1875 , la sévérité moyenne des tribunaux décroît du tiers (par rapport à la période précédente) alors que le taux de condamnation double de façon spectaculaire. Le taux de punition, par contre, n'augmente que de $38 \%$. La sévérité moindre des tribunaux annule ainsi près de $60 \%$ de l'impact de la hausse du taux de condamnation. Il est intéressant de noter à quel point les variations apparemment mini- 
Fig. 3 - Taux de punition produite par la prison commune à Montréal de 1845 à 1913 (en mois/hommes par 10000 h.).

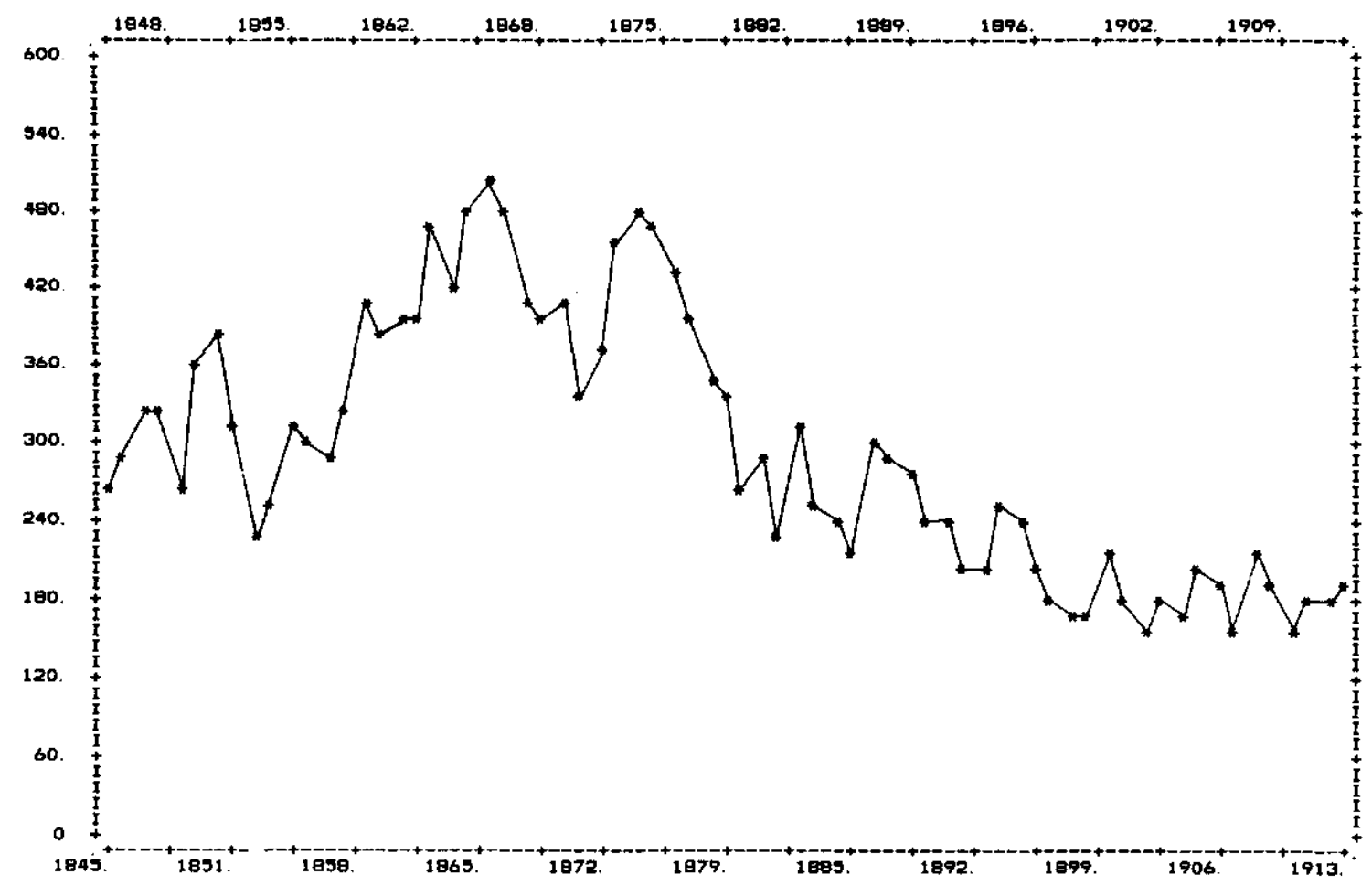


mes de la durée moyenne des sentences infligées par les tribunaux engendrent des effets importants sur la quantité globale de punition imposée. Parce que l'emprisonnement commun et une peine de masse, la plus petite variation de la sévérité des tribunaux se trouve ainsi multipliée.

\section{TABLEAU I}

L'incarcération à la prison commune, Montréal, 1845-1913

\begin{tabular}{cccc}
\hline & $\begin{array}{c}\text { Taux de } \\
\text { condamnation } \\
\text { par 10000 } \\
\text { habitants } \\
(1)\end{array}$ & $\begin{array}{c}\text { Durée moyenne Taux de punition } \\
\text { des sentences } \\
\text { (en mois) }\end{array}$ & $\begin{array}{c}\text { (en mois/homme) } \\
(2)\end{array}$ \\
\hline $1845-1859$ & 186,75 & 1,68 & 309,66 \\
$1860-1875$ & 361,52 & 1,19 & 429,62 \\
$1876-1913$ & 124,60 & 1,89 & 232,17 \\
$1845-1913$ & 193,05 & 1,68 & 294,80 \\
\hline
\end{tabular}

De 1875 à 1913 , le taux de condamnation à la prison commune baisse de $65 \%$ (par rapport à la période antérieure). Les tribunaux sont par contre beaucoup plus sévères (augmentation de $58 \%$ ). Le taux de punition baisse quand même de $45 \%$. En somme, la sévérité majorée des tribunaux annule $20 \%$ de l'impact de la baisse du taux de condamnation. La mesure de la punition du crime se limite souvent à une estimation de sa fréquence relative. Une analyse naïve de l'évolution de la peine commune aurait alors conclu (et interprété subséquemment) des variations brutales de $100 \%$ (pour la deuxième période) et de $65 \%$ (pour la troisième période) alors que les variations sont plus douces (38\% et $45 \%$ respectivement).

Est-on assuré, toutefois, de ce que les variations observées ne soient pas fallacieuses? Le «territoire couvert par la justice criminelle montréalaise a pu se transformer, compte tenu d'un réaménagement du réseau des prisons locales environnant la métropole. Si tel était le cas, on devrait s'attendre à ce que les admissions des prisons locales avoisinant le pourtour encore bien campagnard de Montréal fluctuent en sens inverse de celles de la prison commune métropolitaine, notamment durant les deux 
TABLEAU 2

Analyse de régression de l'évolution de la peine commune, Montréal, 1845-1913

\begin{tabular}{lcccccc}
\hline & Pente & $\mathrm{R}^{2}$ & $\begin{array}{c}\text { Coefficient Constante Moyenne } \\
\text { de } \\
\text { Variation }\end{array}$ & $\begin{array}{c}\text { Pente/ } \\
\text { Moyenne }\end{array}$ \\
\hline $\begin{array}{l}\text { Taux de } \\
\text { condamnation }\end{array}$ & $-2,9$ &, 31 & $44,9 \%$ & 294,9 & 193,05 & $-1,5 \%$ \\
$\begin{array}{l}\text { Sévérité moyenne } \\
\text { des tribunaux }\end{array}$ & 0,007 &, 19 & $19,1 \%$ & 1,41 & 1,68 & $0,4 \%$ \\
$\begin{array}{l}\text { Taux de } \\
\text { punition }\end{array}$ & $-3,24$ &, 42 & $25,8 \%$ & 408,24 & 294,80 & $-1,09 \%$ \\
\hline
\end{tabular}

périodes transitionnelles les plus marquées (1858-1862 et 1879-1883). Les documents sessionnels infirment cependant ce scénario. De 1858 à 1862 , les admissions augmentent non seulement pour la prison commune de Montréal mais également pour celle de Sherbrooke et de TroisRivières. Plus significatif, c'est en 1862 que sont ouvertes en province trois nouvelles prisons, huit d'entre elles se localisant dans les environs plus ou moins immédiats de la métropole. Il est donc très peu probable que la hausse des années 60 résulte d'une sorte de drainage d'un bassin de population géographiquement élargi. Et inversement, de 1879 à 1883 la baisse du taux de punition se vérifie, non seulement à Montréal, mais à St-Hyacinthe ou à St-Jean alors que les populations des prisons de Joliette, Sorel, Trois-Rivières, sont à peu près stables. L'hypothèse d'une fluctuation artificielle de la série peut donc être raisonnablement écartée.

Si la punition commune a bien évolué comme on l'observe à partir des registres d'écrou, plusieurs questions viennent à l'esprit. Pourquoi réprime-t-on plus souvent avant 1875 mais plus sévèrement après? S'agitil de deux «tactiques» différentes de punition? Expriment-elles des préférences de deux générations successives de cette élite "pénale» qui se charge de gérer la punition du crime? Ne témoignent-elles pas aussi d'une transformation des conditions sociales et d'une modification des habitudes de délinquance des classes populaires? Ces interrogations sont bien sûr légitimes, quoique prématurées. Une réponse satisfaisante exigerait une somme de renseignements qui excède largement celle dont nous disposons. Et surtout elles excèdent le terrain assez limité de cette enquête, 
et nous détourne, en fait, de notre intérêt, - la punition elle-même. Car le problème de la punition commune n'a pas encore été abordé comme tel.

\section{LES CLÉMENCES}

Tous les faits n'ont pas été consignés comme il convient. Le dépouillement des registres d'écrou indique qu'une fraction substantielle des individus punis ne purgent pas, en réalité, le principal de leur sentence d'emprisonnement. Nous voudrions montrer comment l'octroi des pardons et la libération par paiement d'amende apparaissent comme deux manières de moduler la peine commune et de fixer le taux de punition effectivement infligée.

Considérez d'abord la petite cohorte annuelle des condamnés qui bénéficient d'une clémence ou d'un pardon c'est-à-dire d'une libération anticipée octroyée par le lieutenant-gouverneur de la province, mais aussi par le magistrat de police, le «recorder" ou les tribunaux. Ces clémences ne couvrent jamais plus de $5 \%$ des condamnés cependant et la moyenne de la série est de $1,8 \%$.

\section{TABLEAU 3}

Clémences accordées aux condamnés de la prison commune, Montréal, 1845-1913

\% des "pardonnés" par

rapport à l'ensemble des condamnés

\begin{tabular}{ll}
\hline $1845-1859$ & 1,62 \\
$1860-1875$ & 1,34 \\
$1876-1913$ & 2,14 \\
$1845-1913$ & 1,84 \\
\hline
\end{tabular}

Les fluctuations qui caractérisent l'octroi des pardons de 1845 à 1913 (voir Appendice C.1) sont irrégulières et aucune tendance globale n'émerge d'une analyse de régression soit du taux de graciés soit du pourcentage des condamnés communs et bénéficiant de ces clémences. Peuton découvrir, pourtant, la fonction de ces pardons dans l'économie globale de la peine commune? Deux faits méritent d'être retenus:

a) Plus la peine est grave, plus les pardons sont fréquents : 9,5\% des condamnés pénitentiaires sont graciés, mais seulement $1,8 \%$ des con- 
damnés communs le sont. Le rôle de la grâce est encore plus éclatant au niveau de la peine de mort.

b) Les variations annuelles des pardons ne sont pas aléatoires, elles tendent à amortir l'impact d'une sévérité acccrue de la peine : lorsque les sentences de peine commune sont plus longues, comme en 1876-1913, le pourcentage de condamnés graciés tend à augmenter. Et inversement, lorsqu'on condamne moins durement mais plus souvent, le pourcentage des graciés diminue, comme dans les années 1860-1875.

Toutefois, la grâce n'est pas le seul ni le plus important mécanisme de stabilisation du taux d'emprisonnement.

\section{L'EMPRISONNEMENT NÉGATIF}

Si les condamnés graciés sont très rares en prison, un deuxième groupe de condamnés, beaucoup plus substantiel, ne purgent pas, eux non plus, le principal de leur sentence. L'emprisonnement commun est principalement un emprisonnement négatif, la plupart des individus incarcérés l'étant par défaut, c'est-à-dire pour n'avoir pas payé l'amende à laquelle ils avaient été condamnés. La situation ne diffère guère au $19 \mathrm{e}$ de celle qui prévaut aujourd'hui. À la fin des années 70 , seulement $36,5 \%$ des détenus de la prison commune dans la province de Québec sont effectivement condamnés à la prison commune, la majorité des condamnés étant incarcérés pour défaut de paiement (Hattem, 1980). La situation est générale au Canada (Commission de Réforme du Droit, 1974) et il s'agit d'une longue tradition comme le montre Delisle (1974) lorsqu'elle analyse la prison montréalaise des années 1930. I n'a pas été possible d'établir de chiffres correspondants à partir des registres d'écrou, pour le $19 \mathrm{e}$ siècle, mais par chance une «irrégularité đans les statistiques pénales de 1913 indique que seulement $28,6 \%$ des individus incarcérés à la prison commune ont été effectivement condamnés à l'emprisonnement ferme, sans possibilité de monnayer leur libération.

Or, parmi le vaste groupe des invididus emprisonnés par défaut, «négativement» en quelque sorte, un sous-groupe retient l'attention. Entre ceux qui s'acquittent sur le champ de leur amende sans être écroués et ceux qui purgeront par defaut leur sentence au complet, s'intercale la cohorte de ceux qui s'acquitteront de leur amende le jour même où ils ont été écroués. Cette cohorte qui représente en moyenne $15 \%$ des condamnés a réorienté l'analyse. Le taux de punition commune calculé plus haut se révèle biaisé à la hausse, les registres d'écrou (et les statistiques selon les périodes), faisant «comme si» - pour simplifier sans doute ces condamnés avaient en fait purgé leur peine carcérale au complet. 
TABLEAU 4

Libération par paiement d'amende après incarcération à la prison commune, Montréal, 1845-1913

\% des libérés par

rapport aux condamnés

\begin{tabular}{rr}
\hline $1845-1859$ & 8,03 \\
$1860-1875$ & 19,47 \\
$1876-1913$ & 16,89 \\
$1845-1913$ & 15,56 \\
\hline
\end{tabular}

L'évolution de la libération par paiement d'amende est résumée dans le tableau 4. L'inspection des données et leur traitement aboutit aux constats suivants :

a) la très grande majorité des individus condamnés à la peine commune s'acquittent sur le champ, sans même être écroués, de l'amende à laquelle ils ont été condamnés. Ce fait a bien été établi aujourd'hui (Hattem, 1980). Faute de statistiques pertinentes, une estimation pour le $19 \mathrm{e}$ siècle à Montréal n'a pu être faite, mais celles de 1913 suggèrent que la situation n'aurait peut-être guère changé depuis 150 ans;

b) l'impact de la libération par paiement d'amende double en importance après 1860 . De 1845 à $1859,8 \%$ des condamnés incarcérés obtenaient une libération anticipée: de 1860 à 1913, 15 à $20 \%$ l'obtiennent;

c) avant 1860 , la libération anticipée par paiement d'amende est étroitement intégrée au comportement global de la justice pénale. Lorsqu'on condamne plus souvent, le pourcentage de libérés par paiement d'amende augmente $(r=, 77)$; par ailleurs le pourcentage de ces libérés décroît lorsque les tribunaux sont plus sévères $(r=-, 68)$. La libération par paiement d'amende amortit le taux de condamnation et renforce le comportement sentenciel des tribunaux;

d) après 1860 , ces intercorrélations qui faisaient système se dissolvent. Le pourcentage de condamnés liberés de prison par paiement d'amende ne semble affecté ni par les variations du taux de condamnation ni par les fluctuations du comportement sentenciel des tribunaux. L'analyse de corrélation pour les périodes 1860-1875 et 1876-1913 aboutit à des valeurs très faibles ou non significatives. 
La punition commune moderne, au Canada et dans un grand nombre de pays occidentaux, présente ainsi une propriété fondamentale: il s'agit dans une large mesure d'un emprisonnement par défaut, d'un emprisonnement négatif. On incarcère ces délinquants qui très souvent n'ont pas été en mesure de payer l'amende à laquelle ils avaient été condamnés. C'est donc par une réflexion sur l'amende et son rôle dans l'économie pénale qu'il semble utile d'amorcer une interprétation des fluctuations observées de la libération anticipée par paiement d'amende.

\section{LE PROBLÈME DE L'AMENDE}

La punition du crime implique généralement un ensemble très diversifié de dispositifs interconnectés. La punition médiévale n'était pas faite seulement de supplices (Hufton, 1981, 32) et la punition contemporaine ne se limite pas à l'incarcération; probation, sentences suspendues, amendes, mesures de sûreté, peine de mort, toutes font partie de l'économie globale de la peine infligée par la justice criminelle. Le rôle de l'amende dans cette économie n'est pas négligeable. Sutherland et Cressey (1966) fournissent quelques estimations intéressantes: à la fin du $19 \mathrm{e}$ siècle les amendes infligées par les tribunaux criminels allemands représentaient $22 \%$ de toutes les peines prononcées, mais $55 \%$ déjà en 1934 ; une évolution similaire caractérise, selon ces auteurs, les économies pénales des justices criminelles française et belge; aux États-Unis $75 \%$ de toutes les peines prononcées sont actuellement des amendes, et en Suède la situation est encore plus marquée. De manière générale l'amende «est de loin la méthode la plus répandue de nos jours pour réagir punitivement contre la criminalité (op. cit., 291).

L'importance de l'amende dans l'économie pénale moderne étant reconnue, doit-on cependant la considérer comme une «autre» peine «à côté», en quelque sorte, de l'incarcération? L'amende n'est pourtant pas une peine aussi générale que l'emprisonnement. Elle sanctionne surtout les infractions sommaires et beaucoup moins les actes criminels. On ne se soucie guère de ce qui semble pourtant inhérent à l'acte criminel, la responsabilité morale de celui qui le commet. Ce n'est que dans la justice criminelle prémoderne, où le droit civil n'est pas différencié du droit criminel, où la punition est en même temps une composition ou une réparation individualisée des dommages subis par la victime, que le règlement monétaire du crime s'applique aux crimes majeurs, le meurtre, le viol ou le vol (Blanshei, 1981 ; Lenman et Parker, 1981).

On ne devrait pas non plus analyser l'amende indépendamment de ses rapports intrinsèques à l'incarcération. La plupart des historiens et 
des bilans consacrés à l'histoire de la peine considèrent d'ailleurs l'incarcération comme la peine spécifiquement moderne du crime, du moins en Occident (Ignatieff, 1981, 158; Hufton, 1981, 34). Pourquoi est-elle devenue si «évidente» et si «normale»? Foucault (1976) suggère qu'en elle s'exprime parfaitement une «nouvelle» manière d'exercer le «pouvoir», d'où son élaboration d'un «modèle», celui de la «discipline». Melossi et Pavanni (1983) définissent l'incarcération comme «privation d'un quantum de liberté proportionnellement et abstraitement fixée a priori», et la considèrent comme spécifique aux économies de marché. Lane (1974) perçoit l'incarcération comme typique des sociétés basées sur une organisation rationnelle du travail qui opère sur une échelle (consommation, production et punition «de masse»). Sellin (1976) est l'un des rares à s'objecter à ces diagnostics: les travaux forcés, longtemps une fonction dominante de la punition du crime, étroitement liée à l'institution de l'esclavage, présupposent un dispositif d' «enfermement» ou d'emprisonnement. Les premiers pénitenciers se structurent d'ailleurs très explicitement autour d'une «productivité pénale». Mais la peine pénitentiaire n'est-elle pas devenue très rapidement improductive et inutile économiquement, du moins en occident, dans les sociétés libérales? Pour qu'une économie de travaux forcés soit rentable sur une grande échelle et de façon stable elle requiert ou bien un régime de terreur (et un taux concomitant de mortalité élevée) ou bien un régime très particulier de micro-société très différencié du régime de vie pénitentiaire (Fogel et Engerman, 1974).

Si l'incarcération est la peine privilégiée de la justice criminelle moderne, il est malaisé de contourner le fait en lui-même bizarre de la prévalence de l'amende. Une première difficulté tient aux points de vue divergents des acteurs de la situation: le juge ayant à «choisin entre deux peines (amende ou prison commune), on en vient à les concevoir comme subsumés sous deux concepts distincts. Mais la perspective de l'accusé est plus juste que celle du pénologue : le condamné ne choisit pas entre deux peines, il paiera plutôt l'amende, s'il le peut, pour éviter l'incarcération et conserver sa liberté, la valeur chérie de la «subjectivité moderne». L'incarcération est la peine qu'on évite en payant l'amende et l'amende le moyen d'éviter ou de se libérer de la prison. Les «équivalences» établies par les tribunaux entre les deux peines, mal étudiées, suggèrent aussi une logique sous-jacente unifiée.

Cette situation n'est pas sans embarrasser les pénologues. Ainsi Hogarth (1971) croit que l'amende est la plus punitive des mesures infli- 
gées par la justice criminelle parce que sa «finalité» exclut la «réhabilitation». Kellens reprend l'argument :

En dehors de son aspect indemnitaire (qui joue un rôle aujourd'hui mineur mais qui pourrait réapparaître) l'amende est à la fois la plus pénale et la plus fiscale des peines. Elle est en effet la peine à l'état pur dans la mesure où son but est de faire suffisamment mal pour dissuader $(1982,142)$.

Une argumentation bizarre qui ne correspond guère à la manière dont l'amende fonctionne réellement dans l'économie pénale nordaméricaine : le montant de l'amende est fixé à un niveau très faible, d'autant plus faible qu'il n'est pas indexé au coût de la vie; la discrétion judiciaire en ce domaine est extrêmement limitée, d'où l'impossibilité de faire correspondre la somme de l'amende à la gravité du crime, à la malice du délinquant ou aux préjudices de la victime (Van den Haag. 1975).

Lorsqu'on spécifie les conditions nécessaires pour que l'amende soit punitive (c'est-à-dire une amende proportionnelle à la gravité du crime, s'exprimant en termes de jours de revenus réels, et imposée à des individus plutôt qu'à des entités corporatives), il devient assez clair, par contraste, que l'amende n'est pas en réalité une peine au sens où l'incarcération l'est. La doctrine libérale du droit criminel n'est guère favorable non plus à ce qu'elle le devienne. Centrée sur le principe de légalité, cette doctrine a été élaborée «avant tout pour assurer le contrôle de certains pouvoirs de l'État (Landreville et Brodeur, 1979, 25) et soustraire, notamment, la punition du crime à des objectifs fiscaux, une caractéristique essentielle, par contre, de la justice criminelle prémoderne (Lenman et Parker, 1981).

Van den Haag montre que l'amende fonctionne plutôt comme unesorte de permis d'opération limitée (la prostitution relève souvent de ce type de contrôle) ou de bail de location temporaire (règles de stationnement public des véhicules). L'amende peut être désagréable mais la peine n'est pas seulement un quantum de désagréments. Elle peut être infligée par un tribunal criminel, mais à moins d'être nominaliste dans un contexte qui n'est pourtant pas ontologique, elle ne se métamorphosera pas pour autant en une punition. L'amende ne serait donc pas proprement punitive, pourtant elle fait partie intégrante d'une économie pénale.

L'interprétation à laquelle se consacre la section suivante ne cherche pas à établir les prémisses d'une théorie générale de l'amende comme sanction juridique. Le problème qu'elle veut résoudre est beaucoup plus limité: pourquoi l'amende permet-elle d'éviter cette incarcération que 
la justice criminelle impose pour mater la petite délinquance? Au lieu de définir a priori ce que "doit être» l'amende, on se demandera plutôt à quoi peut servir l'amende dans une économie pénale centrée sur l'incarcération.

\section{UNE INTERPRÉTATION DES RAPPORTS ENTRE L'AMENDE ET L'INCARCÉRATION}

L'incarcération prive un condamné de sa liberté mais ne s'épuise pas dans cette privation. «La prison, note Foucault, tend à devenir un instrument de modulation de la peine: un appareil, qui, à travers l'exécution de la sentence dont il est chargé, serait en droit de reprendre, au moins en partie, le principe par le biais des libérations conditionnelles, des demi-libertés» $(1976,247)$. La remarque se réfère, dans ce contexte, à la punition pénitentiaire. Mais l'analyse précédente dans sa partie empirique, révèle à quel point la punition commune est elle-même modulée de façon intrinsèque par les clémences et par la possibilité de monnayer son incarcération. Les «bons points» et la liberté conditionnelle paraissent plutôt comme des techniques spécifiques au registre de la peine majeure.

C'est en dépouillant les livres d'écrou et en cherchant à calculer le taux de punition exercée par la prison commune, que s'imposa l'hypothèse d'une analogie structurelle entre la modulation pénitentiaire et la modulation «commune» de la peine. Mais selon quel principe? Donnons, provisoirement, au vocabulaire courant, une portée plus stricte. Une peine est d'autant plus ferme que l'écart entre le taux de punition officiellement infligé et le taux de punition effectivement subie est faible. La gravité de la peine désigne la discontinuité qualitative qui démarque la punition commune de l'emprisonnement pénitentiaire. La sévérité de la peine correspond plutôt aux fluctuations sentencielles internes à chaque registre pénal. Une proposition dérivée de la définition de l'incarcération comme privation modulée de liberté serait la suivante : plus la peine est grave, plus elle est ferme (et moins les condamnés bénéficieront de libération anticipée). Cette hypothèse ne semble pas déraisonnable en soi. Les hypothèses alternatives semblent d'ailleurs mener à l'absurde. Si la peine est d'autant moins ferme qu'elle est grave, il suffirait de commettre le délit le plus sérieux pour bénéficier d'une libértion anticipée maximale. Si la fermeté de la peine était indépendante de sa gravité, une sentence judiciaire prolongée pourrait se transformer selon les circonstances en une détention brève et inversement. 
Les amendes imposées par les tribunaux criminels, tout comme les pardons et les libérations conditionnelles, permettent de moduler et de stabiliser le taux d'emprisonnement. La peine commune étant moins grave que celle qu'on inflige au pénitencier on devrait s'attendre à ce qu'elle soit également moins ferme. Pour l'évaluer on calcula la quantité d'incarcération (en mois/hommes par 10000 h.) qui ne fut pas, en réalité, effectivement subie par les condamnés. À la prison commune, on devait tenir compte des pardons et des libérations obtenues par ceux qui monnayaient leur liberté après avoir été écroués. Au pénitencier et au réformatoire pour délinquants juvéniles, on devait évaluer l'impact des pardons, des «bons points» et des permis de libération conditionnelle. Dans tous les cas on s'est limité aux condamnés provenant de Montréal.

\section{TABLEAU 5}

Importance relative de la quantité d'incarcération évitée par les condamnés montréalais, de 1845 à 1913

\begin{tabular}{lccc}
\hline & $\begin{array}{c}\text { Prison } \\
\text { commune }\end{array}$ & $\begin{array}{c}\text { Réformatoire } \\
\text { pour délinquants } \\
\text { juvéniles } \\
\%\end{array}$ & Pénitencier \\
\hline $1845-1854$ & $\%$ & - & $\%$ \\
$1855-1864$ & 9 & 1 & 9 \\
$1865-1874$ & 14 & 3 & 4 \\
$1875-1884$ & 23 & 16 & 10 \\
$1885-1894$ & 19 & 26 & 9 \\
$1895-1804$ & 18 & 11 & 8 \\
$1805-1813$ & 23 & 11 & 7 \\
$1845-1813$ & 22 & 10 & 6 \\
\hline
\end{tabular}

Comme l'indique le tableau 5, le taux de libération anticipée pénitentiaire a pour effet de diminuer de $7 \%$ en moyenne, la punition infligée par les tribunaux criminels. La peine infligée aux délinquants juvéniles est moins grave : la durée d'incarcération est moins longue et les conditions de vie, moins pesantes. Et on observe, en effet, que l'impact de la libération anticipée est alors plus substantiel qu'au péniten- 
cier, notamment après 1869 , date à laquelle on renonce au modèle d'une "prison réformatrice» au profit d'une «école de réforme».

La peine commune représente en moyenne $50 \%$ de la quantité globale de punition infligée par la justice criminelle montréalaise du $19 \mathrm{e}$ siècle. Mais le plus important des registres du pénal est également le plus bénin. La durée moyenne d'incarcération s'évalue en mois et non en année. Étant la moins grave des peines, on devrait s'attendre à ce qu'elle soit également la moins ferme. On comprend dès lors que l'emprisonnement commun soit, dans une large mesure, un emprisonnement négatif. On incarcère le condamné lorsqu'il ne paie pas l'amende qu'on lui avait imposée. La très grande majorité s'en acquittent sur le champ, sans même être écroués. En outre, $17 \%$ de ceux qui sont emprisonnés parviennent à se libérer presque aussitôt en payant leur amende. On peut donc affirmer que la prison commune est conçue de façon à permettre au grand nombre de l'éviter. L'amende apparaît ainsi comme un modulateur essentiel du taux d'incarcération commune.

Cette interprétation offre certains avantages. Elle permet de sauvegarder la thèse générale de l'incarcération comme étant bien la peine moderne du crime. L'amende n'est pas «une autre peine», mais une propriété dérivée de la gravité de l'incarcération. Elle ne fonctionne pas "comme» une punition mais elle fait partie intégrante de l'économie globale de l'incarcération commune. D'autre part, un problème apparemment insurmontable, celui de l'hétérogénéité qualitative entre l'incarcération et l'amende, peut être ainsi résolu : la punition commune est bien coextensive à celle que produit la prison commune.

\section{DIGRESSION : L'AMENDE A-T-ELLE D'AUTRES FONCTIONS?}

Nous avons jusqu'ici considéré les effets de l'amende sur le taux de punition et adopté, en quelque sorte, le point de vue du condamné. Mais l'amende génère aussi des revenus. Nous ne connaissons pas l'importance de ces revenus ni leur utilisation. Il s'agit là d'un problème à résoudre empiriquement. La punition commune n'est d'ailleurs pas la seule à pouvoir exercer une utilité *économique». Le travail des condamnés pénitentiaires ou même des délinquants juvéniles produit également des revenus ou minimise les dépenses encourues. Le travail pénal n'a jamais été une question secondaire de l'histoire des châtiments (Sellin, 1976).

Nous ne pouvons développer cette thématique comme il conviendrait. Les données ne le permettent pas. Mais on peut déposer quelques 
jalons à titre exploratoire, l'analyse de la peine commune et de son rôle dans l'économie globale de la peine n'étant pas un champ d'étude particulièrement bien quadrillé théoriquement et autrement. La sociologie des organisations et de la déviance (par exemple, Cohen, 1966) a longuement élaboré la thèse du "déplacement des finalités». La raison d'être d'une organisation aurait tendance à se «déplacer», c'est-à-dire à se subordonner à des objectifs «internes» susceptibles d'avantager la vie de ses membres, indépendamment de ses objectifs "externes» officiels. Un corps de police peut augmenter son taux d'arrestation. Mais le taux ne signifie pas nécessairement que le crime se fasse plus féroce ni la surveillance plus habile et insidieuse. Mais qu'elle résulte plutôt du besoin d'obtenir divers bénéfices marginaux pour les membres du corps de police (impact politique local, avantages marginaux divers, etc.) ou encore d'une «tendance» intrinsèque, «bureaucratique», à la croissance. Il n'est pas toujours aisé de donner à cette thèse une portée suffisamment large. Les observations faites sur ce point sont souvent anecdotiques. Il est difficile de s'aventurer dans un secteur où les idées reçues prennent la forme d'une contestation ritualisée et prévisible de toute figure d'autorité.

Il n'y a guère de doute que la punition du crime puisse être assujettie à des fonctions extrinsèques de manière évidente et absolue. Dans une société esclavagiste la punition du crime s'insère dans une économie forcée bien structurée. Dans un régime de terreur la punition du crime s'insère dans une entreprise de liquidation ponctuelle ou massive, dans une économie politique de guerre civile. Dans une société démocratique, la variété libérale qui prévaut en Occident, où la liberté individuelle constitue la valeur prédominante, la punition du crime ne doit ni régler la vie politique ni s'insérer dans la vie économique de la nation. Elle ne doit pas être articulée à une politique de main-d'œuvre au sens où le ministère de l'emploi peut élaborer de tels programmes d'action. La question de l'efficacité relative de la peine à contrôler le crime n'est possible que dans une société où manifestement la peine n'est plus l'instrument délibéré d'une finalité extrinsèque, politique ou économique.

Une deuxième lecture, rapide, des données s'impose, centrée cette fois sur ce que l'analyse empirique déjà présentée ne parvenait pas à assimiler. Ainsi l'impact brusquement doublé des libérations par paiement d'amende dans les années 1860-1875 (et peut-être de l'amende en général) ne s'explique ni par une intensification de la sévérité judiciaire (pour cette période $r=, 004)$ ni par le taux de condamnation $(r=, 10)$. L'analyse de corrélation montre que, contrairement aux pardons, le pourcentage de condamnés libérés par paiement d'amende ne dépend guère, après 1860 , du comportement sentenciel des tribunaux ou des fluctua- 
tions du taux de condamnés (voir Appendice D.1). D'autres facteurs semblent responsables des variations que connaît ce modulateur de la peine commune.

Une hypothèse pourrait s'inspirer de l'analyse de la prostitution de l'époque, un délit exemplaire pour les fins de la discussion puisqu'il est massivement sanctionné par l'amende. La monographie que Brodeur (1983) consacre au "quartier réservé» montréalais des années 1920 - mais celui-ci est mis en place dès 1850 et suscite très tôt ces débats publics ritualisés qui nous sont si familiers - offre une interprétation suggestive. La délinquance des moeurs n'est pas vraiment punie, elle fait surtout l'objet d'un contrôle : l'amende apparaît, en fait, comme une mesure de sûreté dont disposent ceux qui policent les bas-fonds. D'autre part l'amende imposée à la prostitution fonctionne comme une licence d'exploitation qui, à l'époque, s'intégrait harmonieusement dans une économie politique municipale caractérisée par le patronage et la corruption organisée. On ne tolérait pas seulement la délinquance de mours, on en tirait profit également.

\section{RÉCAPITULATION}

Trois propositions assez générales se dégagent de l'analyse menée jusqu'ici :

1) La peine commune représente $50 \%$ du taux global de punition infligée par la société montréalaise à ses délinquants. Une analyse de l'ensemble de l'économie pénale pourrait peut-être établir que plus la peine est sévère, plus la quantité de peine infligée diminue.

2) L'incarcération commune est dans une large mesure un emprisonnement négatif. On incarcère, mais par défaut de paiement d'amende. L'amende n'est pas seulement une autre peine, moins grave, mais une propriété dérivée de la fermeté de la peine. Moins la punition imposée au délinquant est grave, moins elle est ferme. Une interprétation qui sauvegarde la thèse générale de l'incarcération comme la peine spécifiquement moderne du crime.

3) La sévérité de la peine est négativement correlée à sa fréquence relative. Lorsqu'on punit plus souvent, on punit moins intensément. Un mécanisme essentiel de stabilisation du taux de punition. Cette autorégulation stabilisante est-elle une caractéristique générale de la répression du crime?

Un quatrième résultat, qui ne se prête guère à la généralisation, concerne l'évolution de la peine commune au $19^{\mathrm{e}}$ siècle. Le fait marquant 
est sa baisse tendancielle, malgré la modulation exercée par les pardons et l'emploi de l'amende, malgré la stabilisation qu'engendre l'interaction négative entre le comportement sentenciel des juges et le taux de condamnation. Comment expliquer cette baisse tendancielle alors que la société montréalaise s'industrialise et que la métropole s'urbanise (à la veille de la Première Guerre mondiale celle-ci atteint le cap du demimillion)?

Trois sortes d'interprétations peuvent être développées. Une interprétation «sociologique» trouverait dans l'environnement le déterminisme pertinent. Une interpétation «criminologique» chercherait dans le crime que la prison réprime la raison d'être de son évolution. Une interprétation "pénologique» demanderait plutôt à la peine elle-même d'expliquer son propre comportement. Même si l'on se limite à une perspective "pénologique», plusieurs hypothèses peuvent être envisagées. Il existerait, premier scénario, une limite à la capacité institutionnelle d'incarcération des délinquants communs. La croissance démographique de la métropole s'accélérant en fin de siècle, il serait normal, dans ces conditions, que le taux de punition tende à baisser. Cette hypothèse peut être, à notre avis, rejetée pour les raisons suivantes :

a) l'accélération démographique se produit au début des années 1900 seulement; elle ne peut donc pas expliquer la baisse notable que l'on décèle dès 1870 ;

b) la capacité matérielle de punir ne semble pas très contraignante : la cohorte brute des condamnés dépasse le cap des 4000 non seulement en 1911 mais également en 1865 et en 1875 ; le plateau des 2500 condamnés annuels caractérise non seulement la fin des années 1850 mais aussi des années 1880 et au début des années 1900 ;

c) si la capacité institutionnelle exerçait un impact important, on devrait s'attendre à ce qu'une baisse du taux de punition s'accompagne malicieusement d'une hausse du taux de prévenus et réciproquement : en réalité, le taux de prévenus n'est correlé ni au taux de punition ni au taux de condamnation de manière significative et l'analy se de régression montre que, de 1845 à 1913, le taux de prévenus tend à baisser annuellement de $1,5 \%$, une pente légèrement plus marquée que le taux de punition lui-même.

Une deuxième hypothèse miserait sur une extension des méthodes du contrôle du crime. Elle s'inspirerait de la discussion précédente consacrée à la fonction extra-pénale de l'amende. Il est possible qu'en fín de siècle on punisse moins la petite délinquance mais qu'elle fasse l'ob- 
jet d'un contrôle de police plus serré. Cette interprétation ne peut être développée comme il faudrait sans excéder les limites de ce travail. Mais elle mérite d'être retenue.

Une troisième hypothèse interrogerait l'économie globale du pénal. La baisse tendancielle de la peine commune serait compensée par une intensification de l'emprisonnement pénitentiaire. Accaparée par une délinquance devenue majeure, la justice criminelle serait plus tolérante ou indifférente à l'égard de la délinquance mineure.

\section{RÉFÉRENCES}

BERNARD, J.P., P.A. LINTEAU, J.C. ROBERT (1975), la Croissance démographique et spatiale de Montréal dans le premier quart du $19^{e}$ siècle, Rapports et Travaux 1973-1975, Groupe de recherche sur la société montréalaise du 19e siècle, Département d'histoire, Université du Québec à Montréal:

BLANSHEI, S.R. (1981), "Criminal Law and Politics in Medieval Bolognia», Criminal Justice History, An International Annual, 1-30.

BRODEUR, J.P. (1983), «Venus doctrinale», in Chabot M., A. Vidricaire (Édit.), Objets pour la philosophie, Montréal, Éditions Pantoute, 3-52.

COHEN, A.K. (1966), Deviance and Control, Prentice Hall, Englewood Cliffs, 1966.

COMMISSION DE RÉFORME DU DROIT DU CANADA, l'Amende, Document de travail $n^{\circ}$ 6, Ottawa, Information Canada.

DELISLE, D. (1974), le Mouvement de la population de Bordeaux, 19/2-1940, École de criminologie, Montréal, Université de Montréal.

FOGEL, R.W., S.L. ENGERMAN (1974), Time in the Cross : The Economis of American Negro Slavery. Boston, Little, Brown and Co.

FOUCAULT, M. (1975), Surveiller et punir, Paris, Gallimard.

HAMELIN, J., Y. ROBY (1971), "L'évolution économique et sociale du Québec, 1851-1899", in F. Dumont, J.P. Montminy, J. Hamelin (édit.), Idéologies au Canada français, 1850-1900, Québec, Presses de l'Université Laval, 13-25.

HATTEM, T. (1980), Prisons du Québec : prisons des pauvres, Mémoire de maîtrise, École de criminologie, Université de Montréal.

HOGARTH, J. (1971), Sentencing as Human Process, Toronto, University of Toronto Press, 1971.

HUFTON, O. (1981), "Crime in Pre Industrial Europe, International Association for the History of Crime and Criminal Justice Newsletter, 4, 8-35.

IGNATIEFF, M. (1981), *State, Civil Society, and Total Institutions : A Critique of Recent Social Histories of Punishment", Crime and Justice : An Annual Review of Research, 3, 153-192.

KELLENS, G. (1982), la Mesure de la peine : précis de pénologie et de droit des sanctions pénales, Faculté de droit, d'économie et de sciences sociales de Liège.

LANDREVILLE, P., J.P. BRODEUR, (1979), Finalités du système de l'administration de la justice pénale et planification des politiques. Cahier de l'École de criminologie $\left(n^{\circ} 2\right)$, Montréal, Université de Montréal.

LANE, R. (1974), aCrime and the Industrial Revolution : British and American Views», Jourmal of Social History, 7, 3, 287-303. 
LENMAN, B., G. PARKER (1980), *The State, the Community, and the Criminal Law in Early Modern Europen, in V.A.C. Gattrell, B. Lenman, G. Parker (édit.), Crime and the Law. The Social History of Crime in Western Europe since 1500, Londres, European publications, $11-48$.

LINTEAU, P.A., R. DUROCHER, J.C. ROBERT (1979), Histoire du Québec contemporain : de la Confédération à la crise, Montréal, Boréal Express.

MELOSSI, D., M. PAVARINI (1983), The Prison and the Factory. Origins of the Penitentiary System, New York, Barnes and Noble.

SELLIN, TH. (1976), Slavery and the Penal System, New York, Elsevier.

Sutherland, E., D. CRESSEY (1966), Principes de Criminologie, Paris, Cujas.

VAN DEN HAAG E. (1975), Punishing Criminals : Concerning a Very Old and Painful Question, New York, Basic Books. 